\title{
DESIGN OF A VIRTUAL LABORATORY FOR ANALYZINg Nanoscale Magnetic Materials
}

\author{
Wernhuar Tarng ${ }^{1}$, Ya-Hsuein Cheng ${ }^{1}$, Chih-Ming Lin ${ }^{2}$, Chi-Young Lee ${ }^{3}$, \\ Hsin-Hun Liou ${ }^{4}$ \\ ${ }^{1}$ Graduate Institute of e-Learning Technology, \\ National Hsinchu University of Education, Taiwan \\ ${ }^{2}$ Department of Applied Science, \\ National Hsinchu University of Education, Taiwan \\ ${ }^{3}$ Department of Materials Science and Engineering, \\ National Tsing Hua University, Taiwan \\ ${ }^{4}$ Department of Computer Science and Information Engineering, \\ National Central University, Taiwan
}

\begin{abstract}
As the advance of technology, the manufacturing process of materials has moved forward from the scale of micrometer to sub-micrometer and nanometer. Combining nanotechnology and traditional magnetic materials, nanoscale magnetic materials can be created for applications in biomedical examination and therapy as well as data recording to increase the storage space of a computer. The topic of using the magnetic force microscope (MFM) to exam a material's magnetic field distribution can often be found in nanotechnology courses. Due to the cost of equipment and difficulty of its operation, the teacher can only teach students with an instructional video most of the time instead of using a real MFM to conduct experiments. As a result, students may not fully understand its principle and operating procedure. In this study, a virtual MFM laboratory was designed as an app for execution on tablet computers to increase students' learning interest and motivation. Without using expensive equipment, they can understand the magnetic field distribution of materials by using the virtual MFM to examine different samples provided in the virtual laboratory. A teaching experiment was also conducted to compare the learning effectiveness of using an instructional video and the virtual MFM laboratory. The experimental results showed that using the virtual MFM laboratory was more effective than using the instructional video; the questionnaire results also revealed that most students had positive attitudes toward the virtual MFM laboratory and they thought it could enhance their learning interest and motivation.
\end{abstract}

\section{KEYWORDS}

Nanotechnology, Magnetic Force Microscope, Biological Compass, Virtual Reality, Situated Learning

\section{INTRODUCTION}

Nanotechnology is a new technology rising rapidly in recent years [1]. However, in the year 1959, Feynman, a famous American theoretical physicist and Novel Prize winner, gave a speech "There's Plenty of Room at the Bottom" in which he calculated how many Encyclopedia Britannicas could fit on a pin head. He predicted that the development of technology will move forward down to the micro scale. The idea was considered incredible by people at that time and it is now realized by nanotechnology. Nanotechnology is the new hope of economy in the 21st century, and it changes the industrial structure and human lifestyle. Therefore, it is considered as 
The International Journal of Multimedia \& Its Applications (IJMA) Vol.6, No.1, February 2014

the fourth industrial revolution. Some scientists even predict that its impact on human life is greater than that of information technology and semiconductor industry.

Nanometer $(\mathrm{nm})$ is a length measurement unit and $1 \mathrm{~nm}$ equals $10^{-9} \mathrm{~m}$, which is about the length of three to four atoms in alignment. Research on physical and chemical characteristics as well as manufacturing, manipulation and development of equipment for the measurement of materials between the scales of $0.1 \mathrm{~nm}$ and $100 \mathrm{~nm}$ can be called nanoscience or nanotechnology. More precisely, the term nanoscience is used to describe the interdisciplinary fields of science devoted to the study of nanoscale phenomena employed in nanotechnology. Scientists discover that a material at nanoscale may have totally different physical characteristics, e.g., transmittance, electric conductivity and magnetism. Therefore, it will become a new material and that's why nanotechnology is getting more and more important.

The world of nanotechnology is composed of atoms, molecules, macromolecules, quantum dots and macromolecular assemblies, and they are dominated by surface effects such as van der Waals force attraction, electronic charge, etc. Its miracle comes from the quantum and surface effects of nanoscale materials and evolves many important applications. Nanotechnology is an integrated and broad area of study which includes: nanophysics, nanochemistry, nanomaterial science, nanobiology, nanoelectronics, nanomanufacturing, and nanomechanics. Its applications encompass medical science, environmental protection, and energy, and it can also be used to increase the performance of sensors, chemical catalysis, thermodynamics, electricity, physics, chemistry, optics, mechanics and magnetism [2].

Many phenomena in nature are related to the properties of nanostructures. For example, lotus leaves are not stained when growing out of mud because the nanostructure on their surface causes the hydrophobic effect and the self-cleaning effect. The brilliant colors of butterflies change according to the viewing angles because the nanostructure on the wing surface reflects light with a certain wavelength, making the colors change with the direction of incident light. Bees, salmon, pigeons, and green turtles can travel a long distance without losing their way since their biological compasses help them to identify the right direction. These animals have magnetinduced cells at different positions in their bodies, e.g., the front beak of a pigeon, the head of a turtle, and the abdomen of a bee, for providing the function of a biological compass to tell directions under geomagnetic navigation [3].

For physicists, nanoscale materials are too small for observation, but they are too large for chemists to manipulate because hundreds of atoms or molecules are included. In 1981, the scanning tunnelling microscope (STM) was invented by Rohrer and Binning at the IBM Research Laboratory in Zurich, Switzerland [4]. Its basic principle was to use the quantum-mechanical tunnelling effect to measure the nanostructures on material surfaces by using a tiny probe. In 1986, Binning, Quate and Gerber invented the AFM, a modification of STM, by measuring the van der Waals force between atoms to determine the geometry shape on the surface of a sample. It was the most direct tool for measuring nanoscale materials [5]. The magnetic force microscope (MFM) was originated from the AFM in 1987, and it can be used to measure a material's magnetic field distribution [6].

The topic of using the MFM to analyze magnetic materials is often found in nanotechnology courses in colleges. The MFM is expensive and professional equipment such that only the users with a license are allowed to operate it in a laboratory. Due to the limited amount of equipment and difficulty of operation, the teacher can only teach the courses with an instructional video most of the time instead of using a real MFM. As a result, students may not be able to fully understand its principle and operating procedure. In this study, a virtual MFM laboratory was designed based on the virtual reality technology and situated learning theory to increase the learning interest and motivation of students. Without limitation of available equipment or worrying about its damage, 
students can better understand the magnetic field distribution of materials by using the virtual MFM laboratory to conduct some experiments.

Virtual Reality (VR) is a created environment on the computers with human-machine interface to simulate physical presence in places in real or imagined worlds. The users can see, hear and even feel in the created scenes as if situated in the real world, and even interact with avatars or objects in the virtual scenes [7]. Virtual reality can simulate our surrounding environments or the world which can not be seen by the naked eye, and it can even create a space by imagination for users to observe or interact with. Consequently, it has the attractive features of simulated teaching materials, e.g., creating environments not easy to achieve in the real world, providing experimental environments at a lower cost or free of danger, and so on. Besides, virtual reality provides a safe learning environment for students to practice anytime, anywhere and repeatedly such that they can learn from trial and error [8].

Situated learning was first proposed by Brown et al. [9] and its concept is to simulate a realistic or virtual environment to provide learners with learning situations for exploring knowledge by the interaction with real or simulated teaching materials. The learning activity is designed for learners to participate in the process of learning, introspection, investigation and feedback, and the objective is to adaptively develop suitable knowledge and ability for them to apply in the future [10]. The advantages of situated learning include: enhancing students' learning interest, independent thinking and development to solve the problems, and using technologies to support teaching materials for increasing the depth and width of learning, opportunities of practice and cooperation between learners [11].

In this study, a virtual MFM laboratory has been developed to support the National Nanoscience Training Project (NNTP) launched by the National Science Council (NSC) in Taiwan and the objective is to promote the related knowledge of nanotechnology in K12 science education as well as general teaching in colleges. A teaching experiment was conducted to compare the effectiveness of using an instructional video and the virtual MFM laboratory in learning the knowledge of MFM and magnetic materials. The high interaction and instant feedback of the virtual MFM laboratory allows students to become familiar with the principle and operating procedure of MFM as well as the magnetic field distribution of materials, and it is designed to achieve the following learning objectives:

- Understanding the principle of MFM

- Becoming familiar with the operating procedure of MFM

- Being aware of the scanning modes of MFM and their results

- Learning the characteristics of nanoscale magnetic materials and their applications

- Realizing the concepts of biological compass

For educational applications, the teacher may first introduce the principle of MFM and then ask students to enter the virtual MFM laboratory by using a tablet computer for interactive operation. Students can select a sample by following the system direction and conduct experiments to observe its magnetic field distribution. If they make a mistake during operation, the system will provide a verbal or textual reminder to show the right way of doing it. After all tasks have been completed, the students can take the online test to evaluate their learning achievements. This study used the innovative learning technology to enhance students' scientific literacy, research skills and problem-solving ability. Combining virtual reality with the situated learning theory, the virtual MFM laboratory was designed as an app to achieve the goal of exploring magnetic materials. Students can realize the principle of MFM through interactive operation and instant feedback, and become familiar with the operating procedure via repeated practices. A teaching experiment was conducted in this study to investigate whether the virtual MFM laboratory can enhance students' learning effectiveness as well as their learning interest and motivation. 
The International Journal of Multimedia \& Its Applications (IJMA) Vol.6, No.1, February 2014

\section{RESEARCH METHOD}

The design of MFM was originated from AFM by plating a thin film of magnetic material on the pin head of the probe. The MFM uses the magnetic force between the probe and sample as feedback for analyzing magnetic materials and its operation is similar to that of the AFM. When conducting a measurement, the magnetic force between the probe and sample can be derived as $\vec{F}=\mu_{o}(\vec{m} \cdot \nabla) \vec{H}$, where $\vec{m}$ is the probe's magnetic torque, $\vec{H}$ is the magnetic field on the sample surface, and $\mu_{o}$ is the magnetic permeability in the free space. The MFM uses the probe to scan a sample for constructing the magnetic field distribution on its surface by detecting the magnetic force between the probe and samples such as magnetic recording materials [12].

\subsection{System Design}

The development environment of the virtual MFM laboratory is Microsoft Windows 7 with the software Shiva3D and 3ds Max. In addition, the tools of JDK, Android 1.5 SDK, Eclipse and Android Development Tool Plug-in are required. After the system was completed, ShiVa3D's Authoring Tool was used to convert it into the installation file, which was published on Google Play for users to download. The system modules of the virtual MFM laboratory include camera and touch-screen controllers, API programs for user interface control and online test. In addition, the modules of flight simulation, 3-axis accelerometers and collision detection were designed specifically for the motion-sensing game of biological compass.

In this study, the virtual MFM was developed based on the high vacuum scanning probe microscope (SEIKO SPA-300HV) at Nanometer and Material Technology Center, National Tsing Hua University, Hsinchu, Taiwan (Figure 1). This study used 3ds Max to develop the MFM model and Photoshop to create the required texture images. Finally, the VR software ShiVa3D was used to design the user interface and control programs to simulate the operating procedure of a real MFM. The objective of this study is to develop a virtual MFM laboratory for learning how to analyze the magnetic field distribution of materials. The users can become familiar with the principle and operating procedure of MFM after using the virtual laboratory, and understand the concepts of biological compass by playing the motion-sensing game. An online test was also designed for users to evaluate their learning achievements.
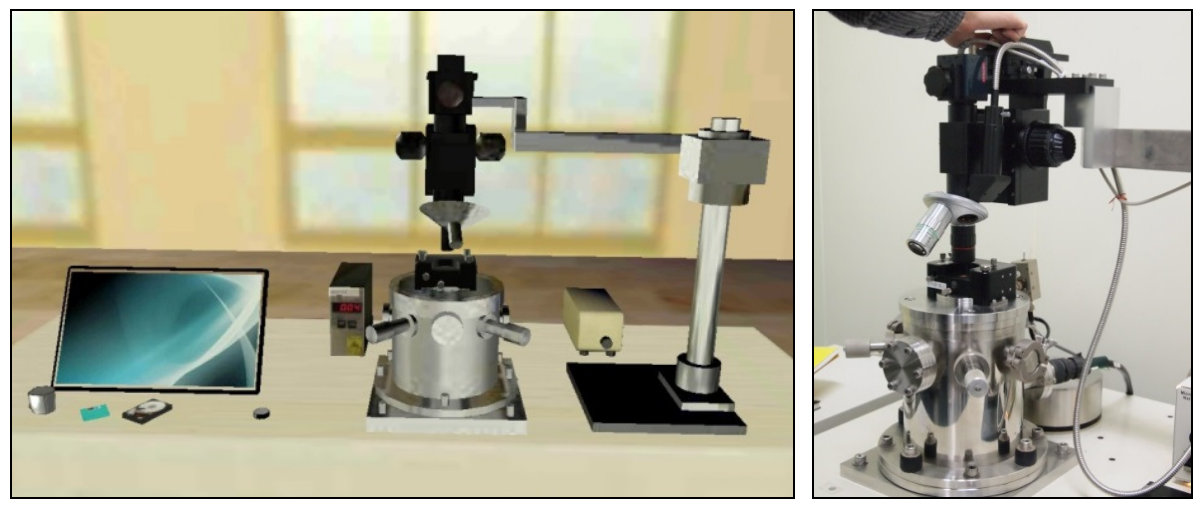

Figure 1. The virtual MFM model (left) and the real MFM (right)

\subsection{Operating Procedure}

Following the operating procedure described below, the users will see the welcome page first when the system is started up. They can click the button 'Introduction' to study the learning objectives and how to operate the MFM. After reading the introductory contents, they can enter 
The International Journal of Multimedia \& Its Applications (IJMA) Vol.6, No.1, February 2014

the virtual MFM laboratory and the verbal prompts will guide them to open the task checklist and perform virtual experiments. If the users are not familiar with the operating procedure, they can click the triangular button on the left to open the task checklist, and the system will direct the users to select any uncompleted tasks. In each step, the item for selection is marked with a red circle for users to know its position easily. The major steps for the operating procedure of MFM include: (1) introduction to the MFM, (2) magnetizing the probe, (3) selecting a sample, (4) installing the laser box and optical microscope, (6) calibrating the laser beam, (7) obtaining the probe's resonance frequency, and (8) performing the first and second scans to obtain results. The system provides three samples (nickel, floppy disk and hard disk) for users to select, and they can complete the experiment easily by following the system direction. After that, (9) the concept of biological compass is introduced, and the users can (10) play the motion-sensing game and (11) take the online test to evaluate their learning achievements.

\section{(1) Introduction to the MFM}

When the users start the system, they can either click the button 'Introduction' to become familiar with the principles and operating procedures of MFM or enter the virtual MFM laboratory directly to conduct experiments. After entering the virtual MFM laboratory (Figure 2), the screen will show its learning objectives first. In the virtual MFM laboratory, the users can slide their fingers on the touch screen to change the perspective, which helps them to see and operate each component of the equipment in the laboratory easily.

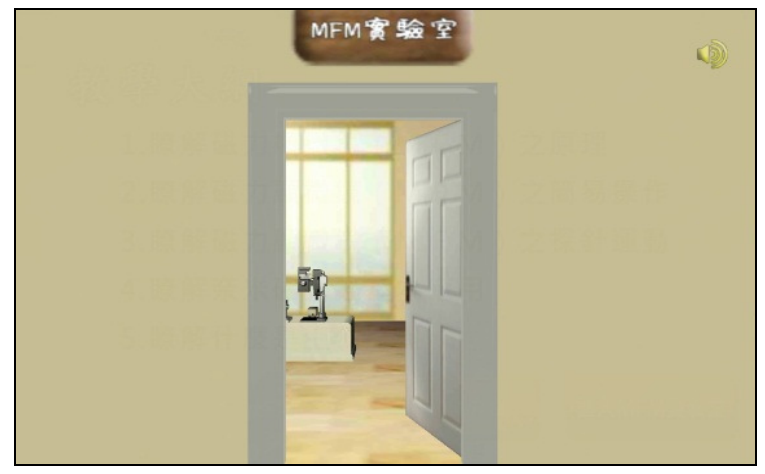

Figure 2. Entering the virtual MFM laboratory

When using the virtual MFM laboratory, the verbal prompts will remind users to click the triangular button on the left to open the task checklist, and they will direct users to select any uncompleted tasks. After clicking a task, an explanatory window will be opened and a verbal prompt will guide them to complete the task. When the task checklist is opened, the users can still change the perspective by sliding their fingers on the screen, which makes it easy to see every piece of equipment in the laboratory. Users can click the triangular button again to close the task checklist, or they can select a component in the laboratory to study its function (Figure 3). If the users select the task 'Online Test' before all the remaining tasks have been completed, a warning message will pop up reminding that they have not completed the assigned tasks yet and thus are not able to take the test temporarily. 
The International Journal of Multimedia \& Its Applications (IJMA) Vol.6, No.1, February 2014

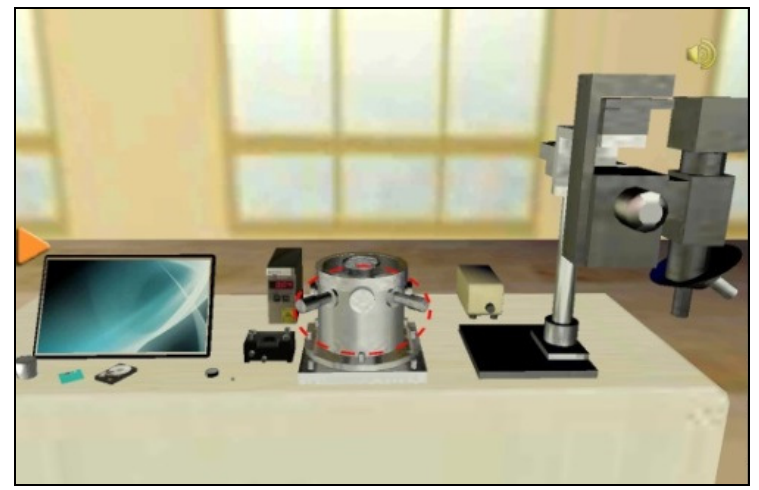

Figure 3. Selecting a component to study its function

\section{(2) Magnetizing the probe}

The basic principle of MFM is similar to that of the AFM except that the MFM uses the tapping mode as well as the lift mode to perform scanning. As a result, the induced force of the probe is the combination of van der Waals force and magnetic force. The van der Waals force is a weak force, so the working distance is closer to the sample; the magnetic force is a strong force, so the working distance is farther. The probe has to be magnetized before scanning, and it is induced only on top of magnetic areas when scanning the sample. By selecting the probe on the table, it is moved to the top of a magnet for magnetization (Figure 4).

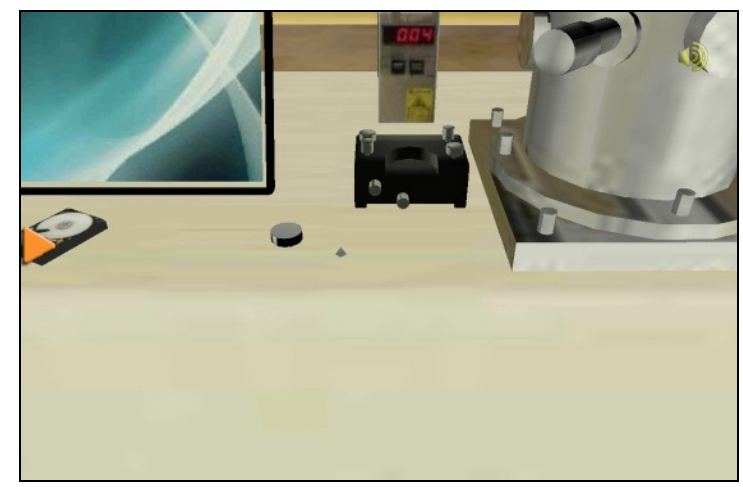

Figure 4. Selecting the probe for magnetization

\section{(3) Selecting a sample}

The virtual MFM laboratory provides three samples for selection, i.e., nickel, floppy disk and hard disk. By clicking a sample on the table, the users will see an introduction to the sample first. They can click the button 'OK' to move the sample to the MFM for scanning (Figure 5) or click the button 'Cancel' if they decide to use another sample. After selecting another sample, it will replace the old one and be moved to the MFM for scanning. 
The International Journal of Multimedia \& Its Applications (IJMA) Vol.6, No.1, February 2014

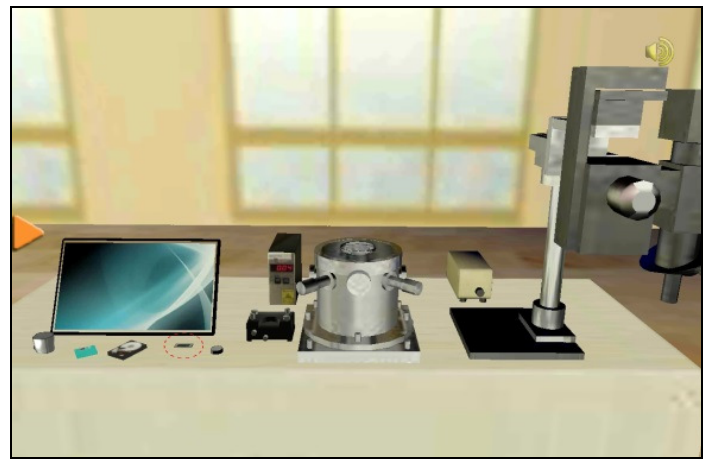

Figure 5. Moving the selected sample to the MFM

\section{(4) Installing the laser box and optical microscope}

To perform experiments on the MFM, the users have to install the laser box and the optical microscope on top of the MFM (Figure 6). The function of laser box is to adjust the position of the probe, and the function of the optical microscope is to observe the reflected laser beam for detecting the probe's position.

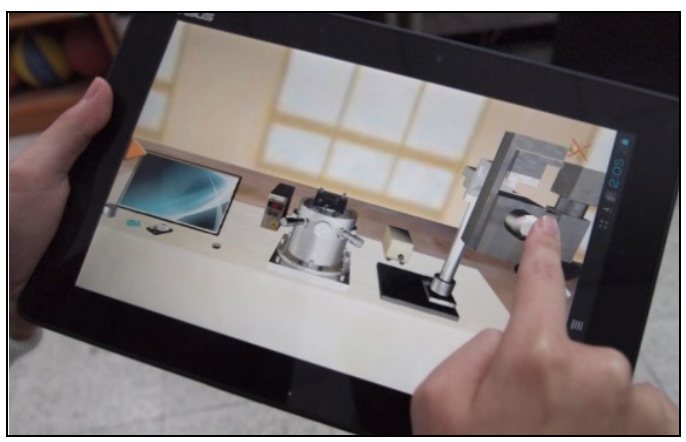

Figure 6. Installing the laser box and optical microscope

\section{(5) Calibrating the laser beam}

When calibrating the laser beam, the users will see an introduction describing why should and how to calibrate the laser beam. The users can start calibration by clicking the buttons to move the laser beam to the pin head of the probe (Figure 7). After the calibration, the probe of MFM has been set to the initial position and is ready for scanning.

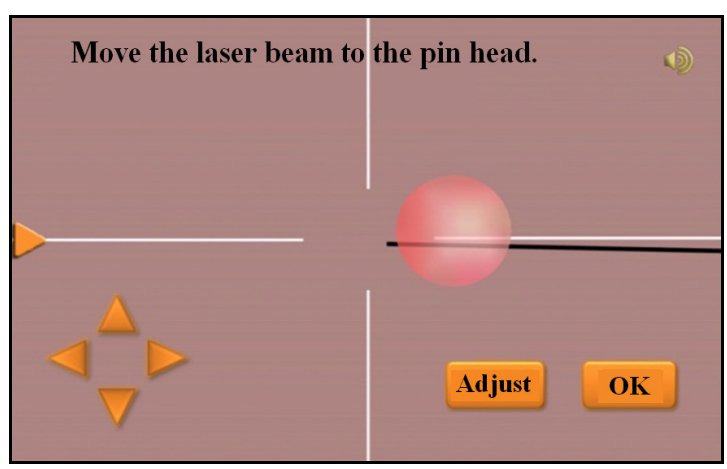

Figure 7. Calibrating the laser beam 
The International Journal of Multimedia \& Its Applications (IJMA) Vol.6, No.1, February 2014

\section{(6) Obtaining the resonance frequency}

To calculate the magnetic force between the probe and the sample accurately, the users have to set the probe's oscillation frequency because it is sensitive to the change of magnetic field on the sample surface. The users can click the button 'Start' to obtain the resonance frequency between the probe and sample, and the screen will display the pulse of the resonance frequency on the screen. The users have to move the frame to encompass the pulse by clicking the button 'Left' or 'Right' to obtain the resonance frequency (Figure 8).

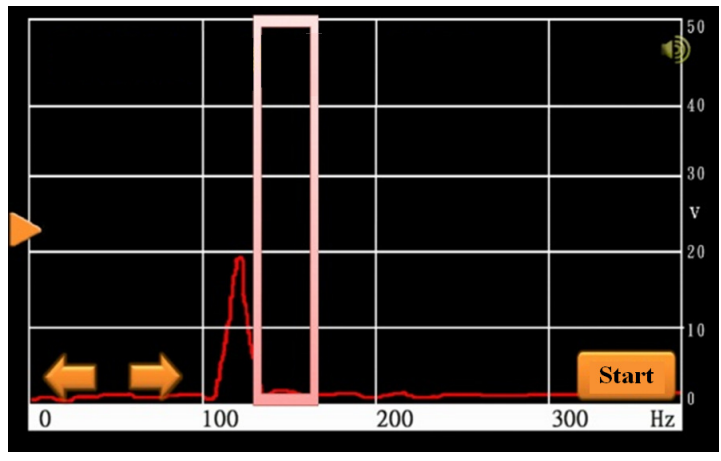

Figure 8. Obtaining the resonance frequency

\section{(7) Performing the scans}

The MFM performs the first scan by the tapping mode to obtain the topographic image of the sample's surface, so the height for scanning is $10 \mathrm{~nm}$ to measure the van der Waals force. The users have to set the correct height before the scan is carried out, or the system will show an error message reminding the users to adjust the height again. The MFM conducts the second scan by the lift mode, and the height for scanning is $50 \mathrm{~nm}$ to measure the magnetic force. After that, the effect of van der Waals force in the first scan is subtracted by the computer and the final result will show the magnetic field distribution of the sample only (Figure 9).

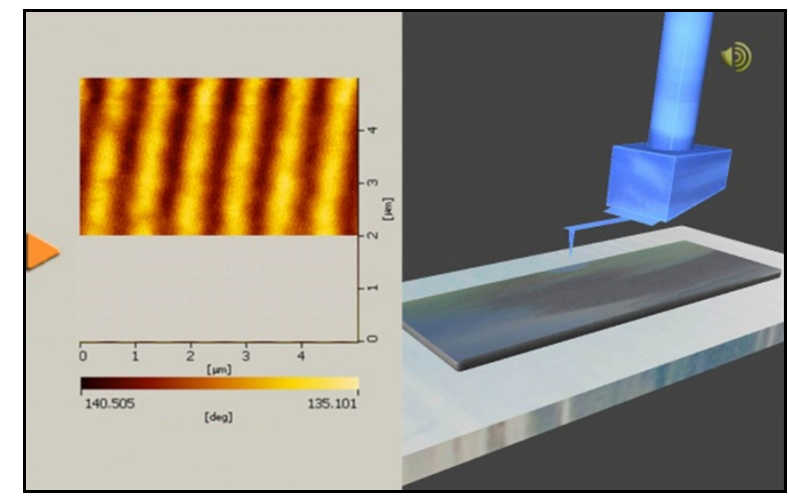

Figure 9. Performing the second scan and the obtained image

\section{(8) Experimental results}

After the second scan, the screen will show the magnetic field distribution of the sample, e.g., the phase images of the floppy disk (left) and hard disk (right) in Figure 10. The distance of 10 magnetic poles for the floppy disk is about $50 \mu \mathrm{m}$, and it is only about $5 \mu \mathrm{m}$ for the hard disk. Therefore, the density of data storage for the hard disk is about $10 \times 10=100$ times that of the floppy disk. As we can see, manufacturing materials with a higher magnetic field density can increase the amount of data recording and thus the storage space of a computer. 
The International Journal of Multimedia \& Its Applications (IJMA) Vol.6, No.1, February 2014
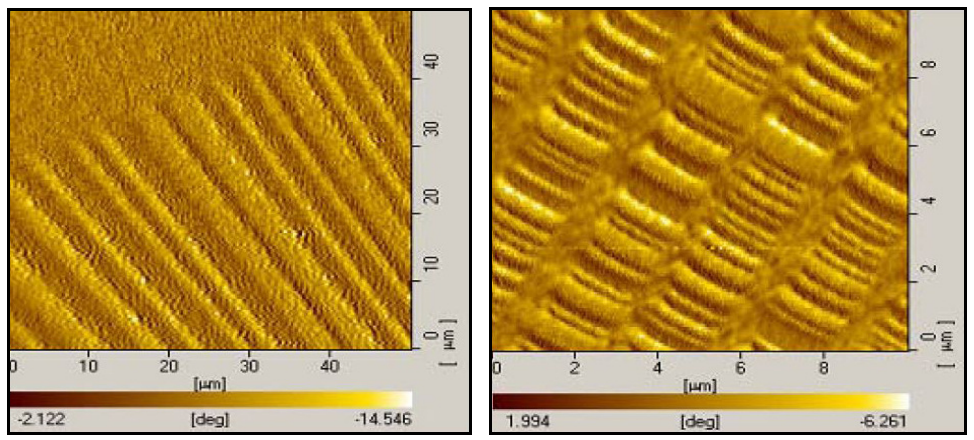

Figure 10. The magnetic field distribution of floppy disk (left) and hard disk (right)

After the experiment, two buttons will show up the screen. By clicking the button 'Repeat the Experiment', the users will enter the virtual MFM laboratory again to perform the experiment. If the users click the button 'Biological Compass', the system will introduce the concepts of biological compass. After that, they can click the button 'Start' to play the motion-sensing game of biological compass.

\section{(9) Biological compass}

Many animals such as bees, salmon, pigeons, turtles, lobsters and dolphins are able to travel a long way without getting lost since their biological compasses can help them to identify the right direction. These animals have magnet-induced cells (or magnetotactic bacteria) at different positions in their bodies, e.g., the front beak of a pigeon, the head of a turtle, and the abdomen of a bee, for providing the function of a biological compass to tell directions under geomagnetic navigation. For example, the left image in Figure 11 shows the magnetotactic bacteria in the front beak of a pigeon and their function to tell directions is enhanced by the collaboration of the pigeon's nervus ophthalmicus and nervus trigeminus.
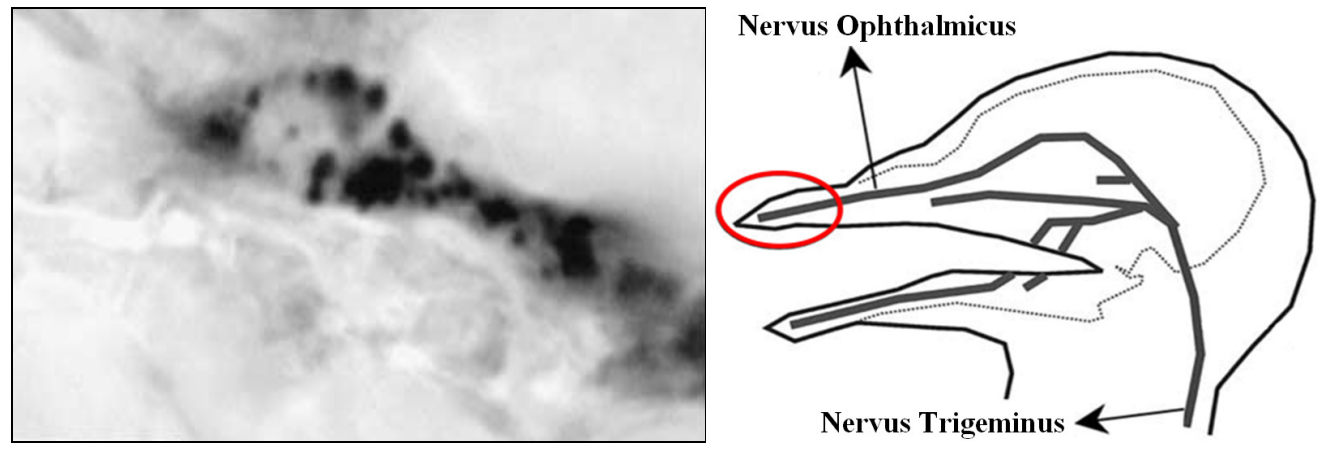

Figure 11. Magnetotactic bacteria in the front beak of a pigeon

\section{(10) Motion-sensing game}

The users can use their hands to incline the tablet computer for the control of flight directions to help the pigeon return home safely (Figure 12). Two kinds of attacks (eagles and thunders) may occur on its way home, and the users can operate the table computer to escape the attacks. After reaching the destination, the system will explain the concepts of biological compass in more detail. The users can start a new game or take the online test to evaluate the achievement in learning the concepts of MFM and magnetic field distribution. 
The International Journal of Multimedia \& Its Applications (IJMA) Vol.6, No.1, February 2014

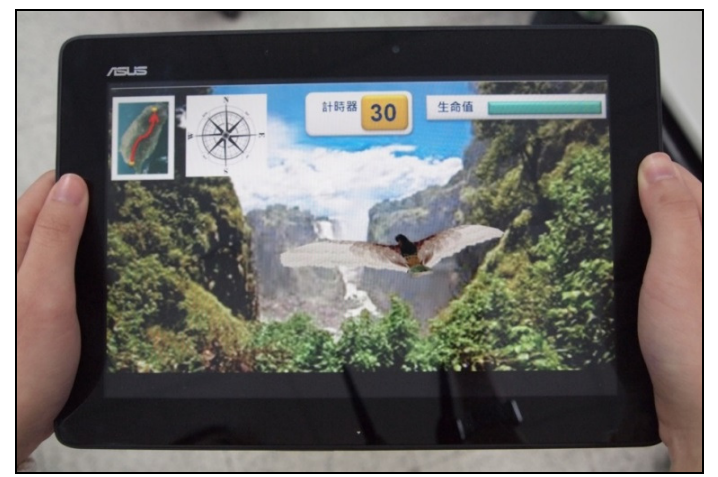

Figure 12. Playing the motion-sensing game of biological compass

\section{(11) Taking the online test}

When the users have completed all tasks on the checklist, the system will request them to take the online test. By clicking the button 'Online Test', the system will display the test item for users to select the right answer. For example, the question in Figure 13 shows: "What is the dominating force in the first scan of MFM?" If the users select (A) van der Waals force, the system will show the message of correct answer; if they select the wrong answer, the system will show the message of incorrect answer and explain the reason.

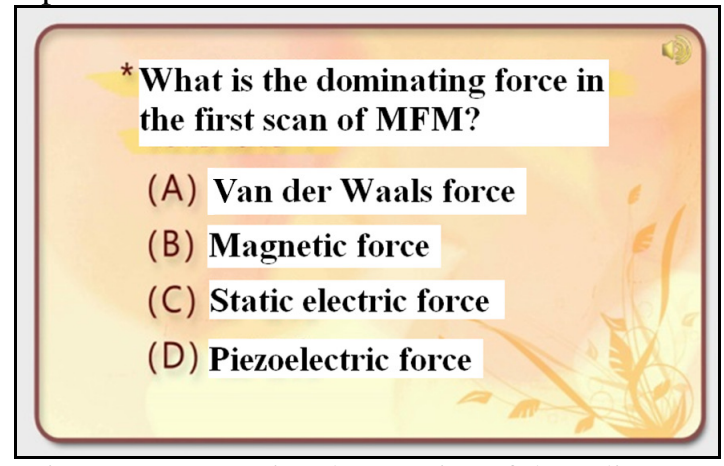

Figure 13. Answering the question of the online test

\section{TEACHING EXPERIMENT}

A teaching experiment was performed to evaluate the learning effectiveness of the virtual MFM laboratory. A questionnaire survey was also conducted to investigate the attitudes of students after using the virtual MFM laboratory to observe the magnetic field distribution of different samples. A number of 38 students were selected from the Department of Applied Science in a university at Hsinch, Taiwan as experimental samples by dividing them into the control group (17 students) and experimental group A (21 students). The control group listened to a lecture and watched an instructional video about the MFM. The experimental group A was given the same lecture and used the virtual MFM laboratory to conduct some experiments. To include the samples of different ages, this study promoted the virtual MFM laboratory at a science camp where the 36 participants (mostly high school students) formed the experimental group B, allowing the comparison of learning achievements between users of different ages.

This study adopted a nonequivalent pretest-posttest design to analyze if there was a significant difference between the learning achievements of different groups. Before teaching activities, all students were asked to take a pretest for evaluating their background knowledge about the MFM and magnetic materials. After the teaching activities, they took the posttest and the experimental 
The International Journal of Multimedia \& Its Applications (IJMA) Vol.6, No.1, February 2014

groups also filled out a questionnaire. The questionnaire survey used in this study was a system satisfaction survey according to users' experiences and it was designed based on the discussion with MFM experts and science teachers. To realize the difference between the two teaching methods, the control group was asked to use the virtual MFM laboratory for 30 minutes at the end of teaching experiment and then filled out an open-ended questionnaire. The flowchart of teaching experiment is shown in Figure 14, and the independent variable, covariance, dependent variable, and control variable for the teaching experiment are listed in Table 1.

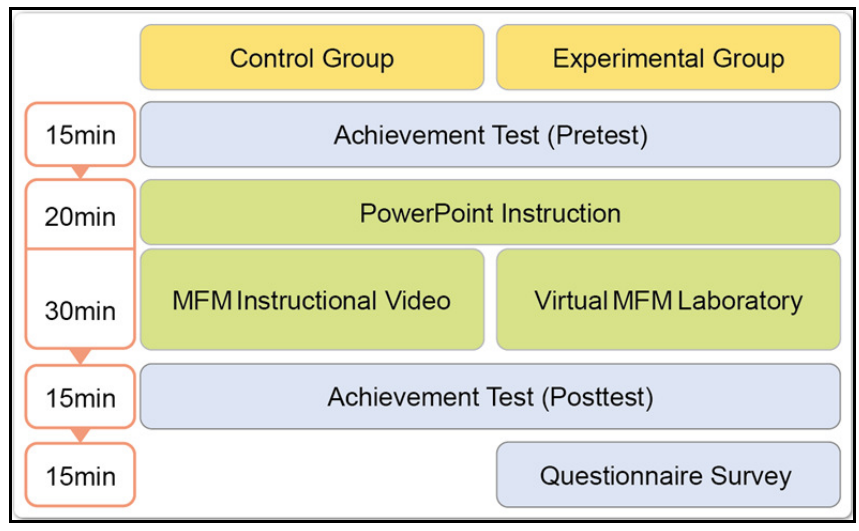

Figure 14. The flowchart of teaching experiment

Table 1. The variables in the teaching experiment

\begin{tabular}{|c|c|}
\hline Variables & Teaching Activities \\
\hline independent variable & $\begin{array}{c}\text { control group: a lecture and an instructional video } \\
\text { experimental groups: a lecture and the virtual MFM laboratory }\end{array}$ \\
\hline covariance & achievement test (pretest) \\
\hline dependent variable & achievement test (posttest) \\
\hline control variable & teacher, teaching time, learning content \\
\hline
\end{tabular}

\subsection{Research Tools}

The research tools used in this study include the virtual MFM laboratory, the instructional video, the achievement test (including the pretest and posttest), and the questionnaire survey, which are described in the following:

- The virtual MFM laboratory

The virtual MFM laboratory is teaching software designed by ShiVa3D, and its contents were designed based on MFM experiments for analyzing magnetic materials. This study combined the VR technology and situated learning theory to develop an interactive teaching environment for users to learn how to use the MFM to analyze the magnetic field distribution of different samples on mobile devices. The system was installed on tablet computers and used by students in the experimental groups.

- Instructional video

The instructional video used for learning by the control group was made by a researcher when conducting an experiment to analyze magnetic materials using the MFM at Nanometer and Material Technology Center, National Tsin Hua University, Hsinchu, Taiwan. The process is similar to the procedure of conducting an experiment using the virtual MFM. 
- Achievement test

The achievement test (including the pretest and posttest) used in this study contained 20 test items which were designed based on the research goal, teaching objectives and MFM learning contents. They were revised according to the suggestions of MFM experts and science teachers.

\section{- Questionnaire survey}

The questionnaire survey used in this study is a system satisfaction survey according to users' experiences and it was designed based on the discussion of MFM experts and science teachers. The questionnaire contained a number of 20 questions (8 items in learning contents, 6 items in interface design, and 6 items in operating experiences) and it adopted Likert's [13] 5-point scale (strongly agree: 5 points, agree: 4 points, neutral: 3 points, disagree: 2 points, and strongly disagree: 1 point). This study used the statistical software SPSS to perform a reliability analysis and the value of Cronbach's Alpha was calculated as 0.95, showing the results had met the standard of high reliability.

\subsection{Learning Effectiveness}

In the teaching experiment, the experimental groups used the virtual MFM laboratory and the control group watched an instructional video for learning the knowledge of MFM and magnetic field distribution of materials. The statistical software SPSS is used to analyze the test results, and Table 2. shows the descriptive statistics of the pretest for the three groups. This study used the Levene test to compare the pretest results to see if any significant differences about the background knowledge of the MFM and magnetic materials existed among these groups; the results show that $\mathrm{F}=4.29$ and $\mathrm{P}=0.02<0.05$ (significant standard), indicating the assumption for the homogeneity of variance is not satisfied. A further analysis reveals that it is caused by the experimental group B, which consisted of high school students with less knowledge about MFM and magnetic materials. After excluding the experimental group B, the analysis is carried out again by comparing the control group and the experimental group A only. The results show that $\mathrm{F}=3.82$ and the significance $\mathrm{P}=0.06>0.05$ and thus the assumption is satisfied.

Table 2. Descriptive statistics of pretest scores for the three groups

\begin{tabular}{ccccc}
\hline Group & Students & Mean & $\begin{array}{c}\text { Standard } \\
\text { Error }\end{array}$ & $\begin{array}{c}\text { Standard } \\
\text { Deviation }\end{array}$ \\
\hline control group & 17 & 56.56 & 3.02 & 12.07 \\
experimental group A & 21 & 57.14 & 1.60 & 7.34 \\
experimental group B & 36 & 50.78 & 2.57 & 14.54 \\
\hline
\end{tabular}

This study used a paired sample T-test to carry out a statistical analysis (Table 3 ) on the pretest and posttest scores of the three groups. For the control group, the $\mathrm{T}$ value is 6.84 and the significance $\mathrm{P}<0.05$, indicating the lecture and instructional video are effective to enhance the control group's learning achievement. For the experimental group A, the T value is 15.41 ; for the experimental group $\mathrm{B}$, the $\mathrm{T}$ value is 3.69 . For both groups, the significance $\mathrm{P}<0.05$, indicating the lecture and the virtual MFM laboratory can enhance their learning achievements effectively as well. 
The International Journal of Multimedia \& Its Applications (IJMA) Vol.6, No.1, February 2014

Table 3. The results of paired sample T-test

\begin{tabular}{cccccc}
\hline Group & Mean & $\begin{array}{c}\text { Standard } \\
\text { Deviation }\end{array}$ & T & $\begin{array}{c}\text { Degree of } \\
\text { Freedom }\end{array}$ & Significance \\
\hline control group & 23.13 & 13.53 & 6.84 & 16 & $0.000^{*}$ \\
experimental group A & 32.14 & 9.56 & 15.41 & 20 & $0.000^{*}$ \\
experimental group B & 12.19 & 18.66 & 3.69 & 35 & $0.001^{*}$ \\
\hline
\end{tabular}

An ANCOVA is conducted to see if there is a significant difference in learning effectiveness between the control group and experimental group A. Before conducting the ANCOVA, the assumptions for the homogeneity of variance and within-group regression coefficients have to be satisfied. The statistical results for the above analysis are described in the following:

- Homogeneity of variance

This study uses the Levene test to verify if there was homogeneity of variance in samples. According to the test results, the value of $\mathrm{F}=3.89$ and the significance $\mathrm{P}=0.056>0.05$. Thus, the assumption is satisfied.

- Homogeneity of within-group regression coefficients

The statistical results show $\mathrm{F}=0.43$ and $\mathrm{P}=0.52>0.05$. As a result, the dependent variable and covariance are not influenced by the independent variable and the assumption of homogeneity is satisfied, so the ANCOVA can be continued.

This study uses an ANCOVA to compare the difference in learning achievements between the control group and the experimental group A (Table 4). Excluding the effect of covariance and dependent variable, the impact of independent variable (the teaching method) is obtained as $\mathrm{F}=9.66$ and $\mathrm{P}<0.05$. A significant difference exists between the learning achievements of control group and the experimental group A. More precisely, the learning achievement of the latter is higher than that of the former after checking the progress made by the two groups in Table 3, indicating that the virtual MFM laboratory is more effective than the instructional video in learning the knowledge of MFM and characteristics of magnetic materials.

Table 4: Statistics results of posttest results for the two groups

\begin{tabular}{cccc}
\hline Group & Mean & Standard Deviation & No. of Students \\
\hline control group & 79.69 & 11.76 & 17 \\
experimental group A & 89.29 & 7.12 & 21 \\
total & 85.14 & 10.44 & 38 \\
\hline
\end{tabular}

\subsection{Questionnaire Results}

This study performs a statistical analysis on the questionnaire results by experimental groups after using the virtual MFM laboratory as a system satisfaction survey (Table 5), where the average score for each evaluation item is denoted by $\mathrm{S}$ in the following discussion. As we can see, the average scores are mostly higher than 4 excepting Question 3 and Question 14, which are related to the speed of tablet computers and their scores are still close to 4 . 
The International Journal of Multimedia \& Its Applications (IJMA) Vol.6, No.1, February 2014

Table 5: The questionnaire as a satisfaction survey of the virtual MFM laboratory

\begin{tabular}{|c|c|c|c|c|c|c|c|}
\hline 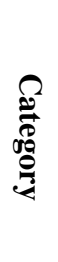 & Evaluation Item & 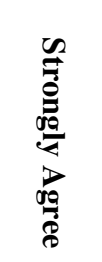 & $\frac{\vec{b}}{80}$ & 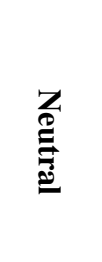 & 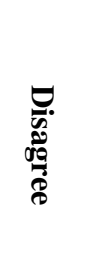 & 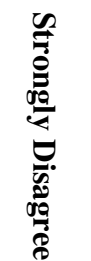 & 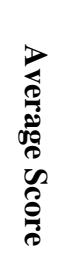 \\
\hline \multirow{8}{*}{ 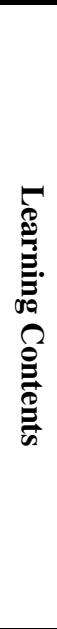 } & $\begin{array}{l}\text { 1. I understand most of the textual descriptions } \\
\text { in the virtual MFM laboratory. }\end{array}$ & $42 \%$ & $43.5 \%$ & $14.5 \%$ & $0 \%$ & $0 \%$ & 4.28 \\
\hline & $\begin{array}{l}\text { 2. The images in the virtual MFM laboratory are } \\
\text { clear. }\end{array}$ & $44.9 \%$ & $43.5 \%$ & $10.1 \%$ & $1.5 \%$ & $0 \%$ & 4.32 \\
\hline & $\begin{array}{l}\text { 3. The virtual MFM laboratory can respond to } \\
\text { my operation quickly. }\end{array}$ & $33.3 \%$ & $29.0 \%$ & $27.5 \%$ & $8.7 \%$ & $1.5 \%$ & 3.84 \\
\hline & $\begin{array}{l}\text { 4. The virtual MFM laboratory provides } \\
\text { adequate prompts. }\end{array}$ & $50.7 \%$ & $34.8 \%$ & $13.0 \%$ & $0 \%$ & $1.5 \%$ & 4.33 \\
\hline & $\begin{array}{l}\text { 5. I understand the steps of operating procedure } \\
\text { after using the virtual MFM laboratory. }\end{array}$ & $44.9 \%$ & $42.0 \%$ & $11.6 \%$ & $1.5 \%$ & $0 \%$ & 4.30 \\
\hline & $\begin{array}{l}\text { 6. The contents of the virtual MFM laboratory } \\
\text { show me the principle of MFM. }\end{array}$ & $44.9 \%$ & $39.1 \%$ & $15.9 \%$ & $0 \%$ & $0 \%$ & 4.29 \\
\hline & $\begin{array}{l}\text { 7. I understand biological compass after playing } \\
\text { the motion-sensing game. }\end{array}$ & $47.8 \%$ & $28.9 \%$ & $17.4 \%$ & $2.9 \%$ & $2.9 \%$ & 4.16 \\
\hline & $\begin{array}{l}\text { 8. I actively completed the tasks and took the } \\
\text { online test. }\end{array}$ & $52.2 \%$ & $34.8 \%$ & $11.6 \%$ & $1.5 \%$ & $0 \%$ & 4.38 \\
\hline \multirow{6}{*}{ 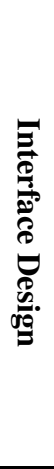 } & $\begin{array}{l}\text { 9. The operation of virtual MFM laboratory is } \\
\text { easy. }\end{array}$ & $43.5 \%$ & $43.5 \%$ & $10.1 \%$ & $1.5 \%$ & $1.5 \%$ & 4.26 \\
\hline & $\begin{array}{l}\text { 10. The text in the virtual MFM laboratory is } \\
\text { clear. }\end{array}$ & $55.1 \%$ & $34.8 \%$ & $10.1 \%$ & $0 \%$ & $0 \%$ & 4.45 \\
\hline & $\begin{array}{l}\text { 11. The verbal prompts in the virtual MFM } \\
\text { laboratory are simple and easy to understand. }\end{array}$ & $44.9 \%$ & $39.1 \%$ & $14.5 \%$ & $1.5 \%$ & $0 \%$ & 4.28 \\
\hline & $\begin{array}{l}\text { 12. I feel intuitive to operate the virtual MFM } \\
\text { laboratory. }\end{array}$ & $47.8 \%$ & $29.0 \%$ & $15.9 \%$ & $5.8 \%$ & $1.5 \%$ & 4.16 \\
\hline & $\begin{array}{l}\text { 13. The visual design of the virtual MFM } \\
\text { laboratory is delightful and consistent. }\end{array}$ & $46.4 \%$ & $29.0 \%$ & $18.8 \%$ & $2.9 \%$ & $2.9 \%$ & 4.13 \\
\hline & $\begin{array}{l}\text { 14. The execution of virtual MFM laboratory is } \\
\text { smooth. }\end{array}$ & $31.9 \%$ & $30.4 \%$ & $24.6 \%$ & $8.7 \%$ & $4.4 \%$ & 3.77 \\
\hline \multirow{6}{*}{ 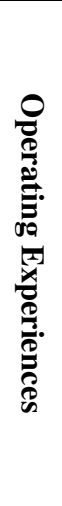 } & $\begin{array}{l}\text { 15. Using the virtual MFM laboratory helps me } \\
\text { understand the major functions of MFM. }\end{array}$ & $34.8 \%$ & $46.4 \%$ & $18.8 \%$ & $0 \%$ & $0 \%$ & 4.16 \\
\hline & $\begin{array}{l}\text { 16. Using the virtual MFM laboratory helps me } \\
\text { understand the principle and operating } \\
\text { procedure of MFM. }\end{array}$ & $36.2 \%$ & $46.4 \%$ & $17.4 \%$ & $0 \%$ & $0 \%$ & 4.19 \\
\hline & $\begin{array}{l}\text { 17. Using the virtual MFM laboratory makes me } \\
\text { want to learn more about biological compass }\end{array}$ & $43.5 \%$ & $33.3 \%$ & $15.9 \%$ & $5.8 \%$ & $1.5 \%$ & 4.12 \\
\hline & $\begin{array}{l}\text { 18. After using the virtual MFM laboratory, I } \\
\text { become more interested in analyzing } \\
\text { nanoscale magnetic materials. }\end{array}$ & $40.6 \%$ & $36.2 \%$ & $21.7 \%$ & $1.5 \%$ & $0 \%$ & 4.16 \\
\hline & 19. The motion-sensing game is fun. & $36.2 \%$ & $37.7 \%$ & $20.3 \%$ & $1.5 \%$ & $4.4 \%$ & 4.00 \\
\hline & $\begin{array}{l}\text { 20. I like to use the virtual MFM laboratory as an } \\
\text { interactive teaching material. }\end{array}$ & $42.0 \%$ & $33.3 \%$ & $24.6 \%$ & $0 \%$ & $0 \%$ & 4.17 \\
\hline
\end{tabular}

\section{(1) Learning Contents}

This section investigates users' satisfaction about the learning contents of the virtual MFM laboratory. Most students thought they could understand the textual descriptions in the virtual 
The International Journal of Multimedia \& Its Applications (IJMA) Vol.6, No.1, February 2014

MFM laboratory $(S=4.28)$ and of which the images were clear $(S=4.32)$. They agreed that the virtual MFM laboratory could respond to their operation in quickly $(S=3.84)$ and they could understand the operating procedure after using the virtual MFM laboratory ( $\mathrm{S}=4.33)$. Besides, they felt that the virtual MFM laboratory provided adequate prompts $(\mathrm{S}=4.30)$ and its contents showed them the principle of MFM $(\mathrm{S}=4.29)$. Also, they could understand the concept of biological compass after playing the motion-sensing game $(S=4.16)$, and they actively completed the tasks and took the online test $(\mathrm{S}=4.38)$.

\section{(2) Interface Design}

This section evaluates users' satisfaction towards the interface design. Most students felt the operation of virtual MFM laboratory was easy $(S=4.26)$ and the text in the virtual MFM laboratory was clear $(S=4.45)$. They thought the verbal prompts in the virtual MFM laboratory were simple and easy to understand $(S=4.28)$ and its operation was intuitive $(S=4.16)$. They considered the visual design of the virtual MFM laboratory was delightful and consistent $(S=4.13)$ and it was executed smoothly $(\mathrm{S}=3.77)$.

\section{(3) Operating Experiences}

This section investigates users' operating experiences. Most students considered the virtual MFM laboratory could help them understand the functions of MFM $(S=4.16)$ and its principle and operating procedure $(\mathrm{S}=4.19)$. After using the virtual MFM laboratory, they would like to learn more about the concepts of biological compass $(\mathrm{S}=4.12)$ and became more interested in analyzing magnetic materials $(S=4.16)$. They thought the motion-sensing game was fun $(S=4.00)$ and they would like to use the virtual MFM laboratory as a teaching material $(S=4.17)$.

\section{Conclusions}

Since the distribution of magnetic field for nanoscale materials is difficult to measure and it is required to use a high resolution microscope or special scientific equipment. This kind of equipment is extremely expensive such that users must be familiar with the operating procedure to avoid damaging it. For ordinary people to learn the characteristics of magnetic materials, they can only search for results on the Internet or go to the library to find the related information in books. Acting in coordination with the NNTP launched by the government of Taiwan, this study developed a virtual MFM laboratory for operation on tablet computers to help students learn how to analyze the magnetic field distribution of nanoscale materials. A teaching experiment was conducted to investigate the learning achievements and attitudes of students after using the virtual MFM laboratory. The results revealed that: (1) the learning effectiveness of virtual MFM laboratory was better than that of the instructional video, indicating the interactive operation under a virtual environment could help students improve their learning achievements; (2) most students gave a positive evaluation towards the learning contents, interface design and user experiences of the virtual MFM laboratory, showing that it was useful to learn the knowledge of MFM and magnetic materials using the virtual laboratory. In addition, the students became more interested in learning the concepts of materials' magnetic field distribution and biological compass after using the virtual MFM laboratory.

This study developed a virtual MFM laboratory to solve the teaching difficulties of using expensive and complicated equipment such as the MFM. It provides knowledge related to nanotechnology through learning situations by allowing students to use the virtual laboratory to analyze magnetic materials. In addition to becoming familiar with the principle and operating procedure of MFM, students can also enhance their knowledge about the magnetic field distribution of nanoscale materials. The use of virtual MFM laboratory is not limited by time or space, and the $3 \mathrm{D}$ visual effects and highly interactive design make learning more interesting and 
The International Journal of Multimedia \& Its Applications (IJMA) Vol.6, No.1, February 2014

effective. As a result, it is a suitable tool for learning nanotechnology in K12 science education as well as general teaching in universities.

\section{ACKNOWLEDGEMENTS}

The authors would like to thank for the financial support of National Science Council, Taiwan under the contract number NSC 102-2120-S-007-008.

\section{REFERENCES}

[1] Chang, L. D. (2003) Nanomaterials, Taipei: Wu-Nan.

[2] Ministry of Education Advisory Office (2008) Introduction to nanotechnology: a supplementary teaching material for high school nano courses, Central and Northern Center for K-12 Educational Development in Nanotechnology, Ministry of Education, Taiwan.

[3] Lee, S. K. \& Wu, C. C. (2004) The symphony of nanotechnology, Taipei: National Taiwan University.

[4] Lu, J. K. (2005) Concepts of nanotechnologies, Taichung: Tsang Hai.

[5] Tsai, Y. C., Shieu, F. S., Lu, F. H. \& Wu, C. M. (2007) Atomic force microscope practical training materials, Taipei: Wu-Nan.

[6] Shu, K. T. \& Chang, L. D. (2004) Nano-compound materials, Taipei: Wu-Nan.

[7] Heim, M. (1993) The metaphysics of virtual reality, New York: Oxford University Press.

[8] Bell, J. T. \& Fogler, H. S. (1996) "Vicher: a virtual reality based educational module for chemical reaction engineering”, Computer Applications in Engineering Education, Vol. 4, No. 4, pp. 285-296.

[9] Brown, J. S., Collins, A. \& Duguid, P. (1989) "Situated cognition and the culture of learning". Educational Researcher, Vol. 18, No. 1, 1989, pp. 32-42.

[10] Lin, Y. S. \& Wang, Y. F. (2009) "Applications of situated learning in curriculums and teaching". Taipei Education, Vol. 96, pp. 69-72.

[11] Shu, H. Y. (1998) "Response of situated learning to educational revolution", Information on Research and Study, Vol. 15 No. 1, pp. 16-24.

[12] Liu, C. C. (2004) A study of magnetic domain structures of Co/Ni thin films by magnetic force microscopy, master thesis, Department of Physics, National Tsing Hua University, Taiwan.

[13] Likert, R. (1932) “A technique for the measurement of attitudes”, Archives of Psychology, Vol. 22, No. 140, pp. 1-55. 